BMJ Paediatrics Open

\title{
Factors influencing maternal decision-making for the infant sleep environment in families at higher risk of SIDS: a qualitative study
}

\author{
Anna Pease, Jenny Ingram, Peter S Blair, Peter J Fleming
}

To cite: Pease A, Ingram J, Blair PS, et al. Factors influencing maternal decisionmaking for the infant sleep environment in families at higher risk of SIDS: a qualitative study. BMJ Paediatrics Open 2017;1:e000133. doi:10.1136/ bmjpo-2017-000133

Received 31 May 2017 Revised 24 July 2017 Accepted 26 July 2017
CrossMark

Centre for Child and Adolescent Health, School of Social and Community Medicine, University of Bristol, Bristol, UK

Correspondence to Dr Anna Pease; a.pease@bristol. ac.uk

\section{ABSTRACT}

Objective Qualitative interviews with mothers of babies at higher risk of sudden infant death syndrome (SIDS) were carried out to understand their views and decision-making process on the infant sleep environment and safe sleep messages.

Design and setting Twenty semi-structured interviews were conducted between February and November 2014 in deprived areas of Bristol, UK. Mothers were asked about their decision-making for the infant sleep environment and safe sleep messages including infant sleep position, co-sleeping, smoking, dummy use, feeding and disrupted routines. The interviews were transcribed, coded and thematic analysis carried out.

Participants Participants were invited based on an algorithm developed in a previous SIDS case control study that identified an increased risk of SIDS from four demographic characteristics: young maternal age, smoking during pregnancy, three or more children, and a measure of deprivation. The presence of three, or more characteristics led to being invited to take part in the qualitative study.

Results Factors influencing mothers' adherence to the safe sleep messages included previous experience and the credibility of the advice given. They described disrupted routines that led to risky scenarios with a belief that occasional risks were acceptable. Where circumstances made following the advice more difficult they found alternative strategies to reduce the risk, including the use of movement monitors, regular checking and a belief that lighter maternal sleep in the presence of a baby was protective.

Conclusions Safer sleep messages should be tailored to fit with the lived realities of mothers, especially those at higher risk. The traditional list of 'do's' and 'don'ts' was not well accepted by this group. Interventions that seek to influence this higher-risk group should acknowledge mothers' own protective instincts and consider their beliefs and understanding behind the safer sleep messages if they are to be effective and encourage this group to change.

\section{INTRODUCTION}

Sudden infant death syndrome, or SIDS, is the unexplained death of a baby for which a thorough investigation has failed to find an

\section{What is known on this topic?}

Young mothers living in deprived areas of the UK have higher sudden infant death syndrome (SIDS) rates than in the general population.

- Efforts to reduce the SIDS rate in this group have had relatively little effect.

The current advice, when adhered to, can greatly reduce the numbers of babies who die.

\section{What this study hopes to add?}

An insight into the night-time care of infants by a group of mothers from deprived areas of the UK with babies at higher risk of SIDS.

- The traditional didactic information-giving model was not sufficient to engage this group of mothers in following safe sleep advice consistently.

- Interventions that target this group will need to consider mothers' own beliefs and understanding behind the safe sleep messages if they are to be effective.

adequate cause of death. ${ }^{1}$ In 2015 , there were 191 unexplained infant deaths in England and Wales, a rate of 0.27 deaths per 1000 live births. ${ }^{2}$ Much higher rates of SIDS cases in the UK in the 1980s (2.3 per 1000 live births, in 1988$)^{3}$ led to a concerted effort to identify risk factors and translate these into parental advice. The initial dramatic 67\% fall in these deaths shortly after the 'Back to Sleep' campaign in $1991^{3}$ has been followed by a further steady $60 \%$ fall in SIDS deaths in England and Wales over the last two decades. ${ }^{4}$

On a population level, there is evidence that implementing risk reduction advice reduces the number of babies who die as SIDS and national campaigns promoting supine sleeping are considered to have contributed to the subsequent dramatic falls in infant deaths in many countries across 
the world. ${ }^{45}$ However, there are also indications that in a residual group of deaths, mostly in poorer families, the risk reduction advice is largely ignored. ${ }^{67}$ There is also a proportional increased risk in unaccustomed sleeping scenarios as deaths in the more usual solitary cot-type environment have declined. ${ }^{8}$ Changes in infant care routines associated with SIDS risk have not been investigated in any detail, and in most SIDS cases in the last 5 years, there is now at least one known risk factor present at the time of death. ${ }^{4}$ It is plausible that interventions that seek to work one-to-one with families at higher risk could make a difference to this situation but if we are to have any meaningful dialogue with those families we first need to expand our understanding of the decision-making processes for the infant sleep environment. This study sets out to understand why some mothers in the UK don't follow the recommended SIDS advice, in particular mothers who are more at risk of suffering a SIDS tragedy.

\section{METHODS}

Eligible participants were identified by purposive sampling using results from a brief face-to-face questionnaire with mothers at health visitor led baby clinics in six deprived areas of Bristol, UK. Areas were defined as deprived if they had Index of Multiple Deprivation (IMD) Scores in the highest quintile ( $\geq 34.18$, most deprived) for England. The questionnaire asked about their knowledge and attitudes to SIDS risk reduction messages. A scoring system to identify families with infants at higher risk of SIDS was developed from the National Confidential Enquiry into Stillbirths and Deaths in Infancy, funded by the Department of Health, ${ }^{9}{ }^{10}$ and has subsequently been used to recruit higher-risk control families in a more recent study. ${ }^{11}$ The four significant predictive factors for higher-risk families included maternal age less than 26 years, smoking during pregnancy, third or subsequent live birth and social class (as determined by occupation) IV (lower supervisory and technical occupations), V (semiroutine and routine occupations) or never employed. Higher-risk families were defined as those having at least three of these four factors. This scoring system showed that $42 \%$ of the SIDS cases could be predicted to occur in this 'higher risk' group which comprised $8 \%$ of the population. ${ }^{9}$

In the current study, occupation was not used to determine social class, instead the full postcode was used to ascertain the IMD Score. Social deprivation in this context was defined as mothers with postcodes in the most deprived quintile for England (highest scoring $20 \%$ ). Mothers with three or four of these factors (young maternal age, smoking, three or more children, and IMD in the most deprived quintile) identified during the completion of an initial quantitative questionnaire were invited to participate in a semi-structured qualitative interview. The quantitative results have been reported separately. ${ }^{12}$
The topics for the interviews were chosen after consideration of the literature and changing epidemiology of SIDS. Interviews were conducted by one female researcher at mothers' own homes and explored decision-making for the infant sleep environment and safe sleep messages including infant sleep position, co-sleeping (defined as an infant sharing a sleep surface with an adult and includes adult beds (bed-sharing), sofas and armchairs), smoking, dummy use, feeding and disrupted routines. The interviews continued until the qualitative research team felt that saturation had been reached and no new information was emerging. With consent, the interviews were audio recorded and then transcribed verbatim, anonymised, checked for accuracy and imported into NVivo V.10. Thematic analysis using a continuous comparative method identified recurrent ideas and patterns within the data. Two transcripts were independently coded by two researchers to enhance rigour by cross checking the coding strategy and early development of themes. During analysis, themes were discussed among the team until a final consensus on the nature and phrasing of key themes and subthemes was reached.

\section{RESULTS}

\section{Participants}

Of the 51 mothers who were invited to take part in an interview, $30(59 \%)$ agreed during the clinic and 20 $(39 \%)$ were interviewed in total, leaving 10 who cancelled before the interview took place. All 10 who cancelled did so via text message and reasons included conflicting appointments, lack of time or a sick child. Differences recorded in the survey were investigated between the mothers who were invited to the interview but declined, mothers who accepted the invitation but cancelled and mothers who were interviewed. There were no significant differences between the groups for maternal or infant age, infant feeding status, parity, IMD Score or smoking status.

Mothers interviewed were aged between 18 and 33 years (mean age of 24 years); babies were aged between 3 weeks and 26 weeks (mean age of 15 weeks).

The interviews ranged in duration from $34 \mathrm{~min}$ to 1 hour and $15 \mathrm{~min}$, (mean duration $55 \mathrm{~min}$ ). The number of mothers with each risk factor present and the number of factors present for each mother are shown in table 1. The majority $(85 \%)$ had three or four risk factors. As recruitment progressed, we identified the need to include more exponents of practices that we were interested in, namely breastfeeding and co-sleeping. Given that these were hard to find in our higher risk population, a decision was taken to invite mothers with only two risk factors present but who had indicated previous experience of breastfeeding and co-sleeping.

Analysis of the audio recordings led to the development of major themes including the influence of previous experience, the credibility of advice and beliefs about 
Table 1 Risk factor profiles of interview mothers

\begin{tabular}{ll}
\hline Risk factor present & $\begin{array}{l}\text { Mothers } \\
\text { interviewed (\%) }\end{array}$ \\
\hline Age under 26 years & $15 / 20(75)$ \\
Smoking at time of survey & $16 / 20(80)$ \\
\hline Three or more children & $11 / 20(55)$ \\
Live in 20\% most deprived area* & $15 / 20(75)$ \\
2/4 risk factors present & $3 / 20(15)$ \\
3/4 risk factors present & $15 / 20(75)$ \\
4/4 risk factors present & $2 / 20(10)$ \\
\hline
\end{tabular}

${ }^{*}$ Determined using full postcode to ascertain the Index of Multiple Deprivation Score-deprived defined as within the most deprived score for $20 \%$ of English wards (areas).

disrupted routines and safe sleep messages that justified alternative, but ultimately unproven ways to reduce the risks of SIDS for their infants.

\section{First theme: previous experience}

Mothers' previous experience had a substantial impact on the decisions they made for their current baby. There was a feeling that if an infant survived in the presence of certain risk factors, this made the risk factors inapplicable or at least optional for future children (box 1). There was a conflict between the evidence that mothers had been given in the form of a safe sleep message, and their own experience of reality. Their previous experience also became associated with a strong sense of 'what works', which related to their ideas of how best to promote the longest amount of sleep for their babies, and themselves. This meant that mothers would sometimes prioritise sleep and settling over safety, especially when these two things were seen to be disrupted (box 2). Mothers' experience from previous children was not always relied on as the best strategy or the one most likely to 'work' for the new baby; finding 'what works' varied for each child. The need to find the most successful strategy often took priority over safe sleep advice, especially when it came

Box 1 P16, age 27 years, five children, smokes, Index of Multiple Deprivation Score: 62.35 (>34.18 is the most deprived quintile)

'Apparently they're supposed to sleep on their back. But to be fair I don't really... because l've got five I just do what I want to do, because I just think to myself as long as my child's happy and sleeps fine, [...] so I think well it worked for the last ten years so I just think like l'll take advice from people, but I won't necessarily do what they're telling me l'm supposed to do, because just think to myself well hang on, I've lived on my own since I was 14, and I know what I'm supposed to. Because l've been a mother since I was 17, I kind of like... I'm like 27 now, so it's like ten years of doing what l've been doing, and it's not like it's been one child. If it was one l'll probably take advice more easily, but I think to myself because it's been five I think well you just know.'
Box 2 P12, age 25 years, one child, smokes, Index of Multiple Deprivation Score: 25.51

.everyone tells you she's got to be there for six months (in the parent's bedroom), and it didn't work for us. She was there for four weeks, um, we were waking each other up, because my partner he works 12 hours shifts as well, it was waking him up, it was waking her up. It was stressing me out because he was next to me, and he never said nothing but I was feeling panicky, like l've got to hurry up because $M$ has got to go to work, not that he would ever say anything like that. She was waking up when we were moving, we were up when she was moving.'

to decisions about infant sleep position and co-sleeping (box 3).

\section{Second theme: credibility of advice}

Mothers felt the style of safe sleep advice was inappropriate, with one mother describing it as 'condescending' and another as being 'lectured'. Mothers said they felt didactic approaches gave little time for absorbing the information or asking questions (box 4). Some mothers described how health professionals had not taken the time to discuss safe sleep, as the mother already had children and it was taken for granted that they would have heard the advice before (box 5). Mothers often described pressure from health professionals to comply with safe sleep messages, and this was connected to a lack of understanding as to why safe sleep messages are important (box 6). In some descriptions, mothers talked about suffocation as a reason to follow safe sleep advice, which for them made sense. Where there was a logical physiological link between advice and risk, mothers were much more open to following the advice, for example, making sure the baby's head could not be covered by tucking the blankets under the armpits and placing baby at the bottom of the cot were both widely mentioned in the interviews and mothers followed this advice without any issues.

Mothers talked about lists of 'do this' and 'do not do this' as unhelpful in helping them make decisions, stating that they would prefer a more individual approach using friendly conversations with people they trust.

\section{Third theme: beliefs about disrupted routines}

Most mothers talked about infant routines, whether they were for feeding, settling or sleep. Mothers used routines to cope with busy schedules and felt that they benefited both the parents and the child. While

Box 3 P1, age 23 years, three children, smokes, Index of Multiple Deprivation Score: $\mathbf{4 0 . 0 0}$

'I just did like, with all of them I just did whatever they were most comfortable at. I didn't care what the proper way was, I did whatever way they were best.' 
Box 4 P4, age 18 years, one child, smokes, Index of Multiple Deprivation Score: $\mathbf{4 3 . 3 1}$

'Yeah, if they just talked to you like have a normal conversation talking about, 'Oh like yeah well we don't advise you to do this, but maybe instead of doing this you can do that,' giving you alternatives or what you could do, instead of just like saying, 'No you can't do this, you can't do that.' Sort of like they were demanding you don't do it all like, do you know what I mean? But eventually someone will rebel against it and they will tell them, 'Well not being funny l'll do what I want, it's my life, it's my body,' do you know what I mean? But they just... I think they need to slow it down slightly, give you a chance to take in the information and allow you to ask the questions.'
Box 8 P2, age 26 years, three children, smoke-free, Index of Multiple Deprivation Score: $\mathbf{4 0 . 0 0}$

'As I said, on the weekends, you know, we both have a bit of slouch, and we might fall asleep on the sofa or something.'

Box 9 P15, age 18 years, one child, smokes, Index of Multiple Deprivation Score: $\mathbf{4 0 . 0 0}$

'It's only like the odd time. Normally I just put him back in his bed after, and then he goes back asleep in there. But the odd I'm like oh no, just go to sleep (in my bed). But I don't tend to do it very often just in case.

situations as inevitable and acceptable as long as it didn't become usual practice (box 9).

Fourth theme: beliefs about alternative strategies for reducing the risk

Where mothers were unconvinced or dissatisfied with the evidence available to them, they provided their own strategies for reducing the risk of SIDS. These strategies broadly fell into three groups: using breathing/ movement monitors, checking the baby frequently and relying on a perceived maternal increased awareness during sleep.

Breathing or movement monitors comprise a sensor pad placed under the baby's bedsheet and detect sound and/or movement. Any significant gaps in sound and/ or movement trigger an alarm. Two mothers talked about their experience of using breathing monitors, one who found it reassuring and gave them peace of mind (box 11), the other mother found the monitor induced anxiety, especially when the alarm went off falsely. Both mothers who had experience of using these monitors did so with the intention of providing

Box 10 P3, age 24 years, three children, smoke-free, Index of Multiple Deprivation Score: 41.83

'And sometimes, you do, like nod off, and like, like, you wake up, and you think like... When I was doing, like, the bottle feeding and stuff in the middle of the night, I just, like, used to feel myself, like, feeding him and just going like that, and like trying to wake myself up. So, like, sometimes you just don't even realise, but, yeah, it is a bit worrying.'

Box 11 P3, age 24 years, three children, smokefree, Index of Multiple Deprivation Score: $\mathbf{4 1 . 8 3}$

'That's how I felt. Like, 'Right, that's in there. He's okay. If anything happens, it'll go off.' That's what I kept thinking, so in that way it kind of just reassured me that everything would be alright.' 
Box 12 P3, age 24 years, three children, smoke-free, Index of Multiple Deprivation Score: $\mathbf{4 1 . 8 3}$

I'd have my head in his Moses basket, because it was right at the side of me. And my face would be in the Moses basket. And I'd constantly like look at him, and see if his chest was moving up and down. Or I'd put my hand on him to check if he was okay.'

Box 13 P13, age 25 years, two children, smokes, Index of Multiple Deprivation Score: 69.65

'When I've got a baby in my arms (and asleep) for some reason, I don't know why, I'm half and half, and I can hear things, and I know that she's fine. If she moved on me I can feel it because I'm half and half.'

an extra layer of protection for their babies, as they had expected the breathing monitor to provide protection, rather than just superficial reassurance.

The use of monitors was similar to the use of checking as a risk reduction strategy. Many mothers talked about constantly or frequently checking their babies while they slept, suggesting that this checking had a preventative element to it. Checking the baby's breathing was a way of knowing that they were alright; that whatever situation they were in was safe since they could see that the baby was still breathing (box 12).

Mothers also talked about an increase in awareness during sleep when in close proximity to their babies. In the words of one mother it was called the 'mum sleep'. Several mothers talked about this awareness as a protective factor; they described it as not being in a deep sleep, still aware of where the baby was and would wake up if the baby moved (box 13). One mother described it as a 'sixth sense' and a few of the mothers relayed stories from their own mothers of this being a known phenomenon for mothers.

\section{DISCUSSION}

Major findings

The aim of these interviews was to investigate influences on maternal decision-making for infant sleep. The findings demonstrate the complexity of how mothers navigate these decisions, varying between families, with different children and even across each night. Mothers let their own previous experience guide them and prioritised sleep and settling over safety on occasions. Mothers found didactic approaches to advice unhelpful, and wanted to know why and how these messages keep babies safe. Mothers in this study coped with the stress of looking after a young baby by going against the advice every now and then and felt that this was normal and justified.

Mothers used alternative strategies to reduce the risk, which were more about helping them feel like they were protecting their infants; they used checking and monitoring and relied on a 'special kind of light sleep' to alert them to any problems with their babies.

Overall, the mothers who took part in these interviews were all aware of the decisions they had made and could account for why they had done the things they did. There were no examples of negligence or wilful ignoring of the safe sleep advice, rather detailed accounts of how mothers have navigated the complexity inherent in looking after young babies without much support. The current approach of advice giving by the midwife or health visitor for reducing the risk of SIDS appears to have limited effectiveness with this group of mothers. Providing mothers with a list of messages may encourage this group to pick and choose which messages they can most easily follow and ignore the rest with the notion that following some, most of the time will offer enough protection.

\section{Comparisons with other studies}

In a recent UK study comparing white British and Pakistani families ${ }^{7}$ with regard to infant care practices, the findings indicated that without clarity on the hierarchy of importance of safe sleep messages, mothers 'trade off' which they would follow. Similarly, the mothers in the present study found ways to feel that they had maintained safety for their babies without having to follow the recommended advice.

Changes to the usual routine (eg, family trips, people visiting, etc) have been shown to increase the risk for SIDS. ${ }^{9}$ The mechanisms behind this increase in risk are not clear, although the potential impact of changes in care practices suggest changes in the infant's sleep environment or social disruption may play just as important a role as social deprivation in these deaths.

Qualitative studies from the USA tend to focus on African-American populations, which have the highest SIDS rates. ${ }^{13-17}$ Cultural differences aside (African-American mothers are more likely to share the bed with their infants), these studies highlight some similarities with the present study findings, including prioritising infant comfort and prolonged sleep. ${ }^{18}$ In a study with adolescent mothers in the USA, the authors report how maternal instincts and information from mothers' mothers overruled the safety advice given to them by health professionals. ${ }^{19}$ Similarly, the mothers in our study made it clear that they needed credible sources of advice that worked with their own instincts, rather than against them. Our interpretation is that mothers do not perceive themselves as prioritising infant comfort over safety, as was suggested in the study with teen mothers ${ }^{19}$ but rather that they prioritise infant comfort over a small perceived risk of a very rare event. Another US study ${ }^{20}$ reported that the mothers talked about a maternal awareness of the baby during sleep, similar to the 'mum sleep' described in the current study.

\section{Strengths and limitations of the study}

A strength of this qualitative analysis is its focus on a higher-risk population. This population is often seen as 'hard 
to reach'; however, for the purposes of this research it was possible to make contact via baby clinics led by health visitors. Having first completed a brief quantitative survey with the researcher it was possible to discuss what would be involved in a home interview and who would be coming to their home. A weakness, as with much detailed qualitative work, is the limited number of mothers that can be recruited in the time allowed and how this generalises to the wider population of high-risk families. Detailed studies such as ours are necessarily small and thus cannot determine the prevalence of these attitudes and beliefs in a wider population.

\section{CONCLUSIONS}

There was no evidence that these mothers with infants at higher risk of SIDS neglected to think about the risks for SIDS or intentionally chose to go against current advice. Faced with challenging night-time decisions and a set of beliefs that made the 'rules' seem less important, many of these mothers were putting their infants at increased risk of SIDS via the sleep environment. However, the use of alternative strategies revolved around the principle of mothers' desire to be a responsive carer and they saw this as a key part of protecting their babies. This protective desire was described in terms of maternal instinct and could be an influential tool during conversations with health professionals about safe sleep.

Conversations with families that focus on the individual circumstances and endeavour to elicit their influences, beliefs and gently challenge their own instinctive views on optimal protective strategies may be necessary to support mothers with making safer infant care decisions. Providing families with more concrete information about why the risk reduction messages are important may also help.

Contributors The study was conceptualised by the research team: AP, JI, PJF and PSB. The research team was involved in designing the study and applying for ethical approval. AP conducted the interviews and analysis, Jl provided guidance and support with coding and the continuous comparative analysis process. The research team together reached a consensus on the final themes. AP wrote the paper with input from JI, PJF and PSB.

Funding The Lullaby Trust; grant number 267.

Competing interests None declared.

Ethics approval West Midlands NHS Research Ethics Committee; ref: 13/ WM/0403.

Provenance and peer review Not commissioned; externally peer reviewed.

Open Access This is an Open Access article distributed in accordance with the Creative Commons Attribution Non Commercial (CC BY-NC 4.0) license, which permits others to distribute, remix, adapt, build upon this work non-commercially, and license their derivative works on different terms, provided the original work is properly cited and the use is non-commercial. See: http://creativecommons.org/ licenses/by-nc/4.0/

(C) Article author(s) (or their employer(s) unless otherwise stated in the text of the article) 2017. All rights reserved. No commercial use is permitted unless otherwise expressly granted.

\section{REFERENCES}

1. Beckwith JB. Defining the sudden infant death syndrome. Arch Pediatr Adolesc Med 2003;157:286-90.

2. Office for National Statistics. Unexplained deaths in infancy. 2015. England and Wales, 2017.

3. Mitchell EA. The changing epidemiology of SIDS following the national risk reduction campaigns. Pediatr Pulmonol Suppl 1997:16:117-9.

4. Fleming PJ, Blair PS, Pease A. Sudden unexpected death in infancy: aetiology, pathophysiology, epidemiology and prevention in 2015 . Arch Dis Child 2015;100:984-8.

5. Frank SJ, Covington TM. "Back to sleep" campaign a success. SIDS rates drop due to information, education. Mich Med 1998;97:22-3.

6. Ford RP, Nelson KP. Higher rates of SIDS persist in low income groups. J Paediatr Child Health 1995;31:408-11.

7. Crane $D$, Ball HL. A qualitative study in parental perceptions and understanding of SIDS-reduction guidance in a UK bi-cultural urban community. BMC Pediatr 2016;16:23. 23 Online.

8. Li DK, Petitti DB, Willinger M, et al. Infant sleeping position and the risk of sudden infant death syndrome in California, 1997-2000. Am J Epidemiol 2003;157:446-55.

9. Fleming $\mathrm{P}$, Blair $\mathrm{P}, \mathrm{Bacon} \mathrm{C}$, et al. Sudden unexpected deaths in infancy: the CESDI-SUDI studies, 1993-1996: Stationery Office Books, 2000.

10. Fleming PJ, Blair PS, Bacon C, et al. Environment of infants during sleep and risk of the sudden infant death syndrome: results of 1993-5 case-control study for confidential inquiry into stillbirths and deaths in infancy. Confidential Enquiry into Stillbirths and Deaths Regional Coordinators and Researchers. BMJ 1996;313:191-5.

11. Blair PS, Sidebotham P, Evason-Coombe C, et al. Hazardous cosleeping environments and risk factors amenable to change: casecontrol study of SIDS in south west England. BMJ 2009;339:b3666.

12. Pease AS, Blair PS, Ingram J, et al. Mothers' knowledge and attitudes to sudden infant deathsyndrome risk reduction messages: results from a UK survey. Arch Dis Child 2017. Online.

13. Hauck FR, Tanabe KO, Moon RY. Racial and ethnic disparities in infant mortality. Semin Perinatol 2011;35:209-20.

14. Oliva G, Rienks J, Smyly V. African American's awareness of disparities in infant mortality rates and sudden infant death syndrome risks. J Health Care Poor Underserved 2010;21:946-60.

15. Schoendorf KC, Hogue CJ, Kleinman JC, et al. Mortality among infants of black as compared with white college-educated parents. $N$ Engl J Med 1992;326:1522-6.

16. Spiers PS, Guntheroth WG. The black infant's susceptibility to sudden infant death syndrome and respiratory infection in late infancy. Epidemiology 2001;12:33-7.

17. Unger B, Kemp JS, Wilkins $\mathrm{D}$, et al. Racial disparity and modifiable risk factors among infants dying suddenly and unexpectedly. Pediatrics 2003;111:e127-e131.

18. Ajao TI, Oden RP, Joyner BL, et al. Decisions of black parents about infant bedding and sleep surfaces: a qualitative study. Pediatrics 2011;128:494-502.

19. Caraballo M, Shimasaki S, Johnston K, et al. Knowledge, Attitudes, and risk for sudden unexpected infant death in children of adolescent mothers: a qualitative study. J Pediatr 2016;174:78-83.

20. Herman S, Adkins M, Moon RY. Knowledge and beliefs of AfricanAmerican and American Indian parents and supporters about infant safe sleep. J Community Health 2015;40:12-19. Online. 